\title{
Pear Blister Canker Viroid: Host Range and Improved Bioassay with Two New Pear Indicators, Fieud 37 and Fieud 110
}

\author{
J. C. Desvignes, D. Cornaggia, and N. Grasseau, CTIFL, Lanxade Center, BP 21, F-24130 La Force, France; and \\ S. Ambrós and R. Flores, Instituto de Biología Molecular y Celular de Plantas (UPV-CSIC), Camino de Vera 14, \\ Valencia 46022, Spain
}

\begin{abstract}
Desvignes, J. C., Cornaggia, D., Grasseau, N., Ambrós, S., and Flores, R. 1999. Pear blister canker viroid: Host range and improved bioassay with two new pear indicators, Fieud 37 and Fieud 110. Plant Dis. 83:419-422.

An investigation was conducted to improve the biological detection of pear blister canker viroid $(\mathrm{PBCVd})$, which over a period of 2 to 3 years induces pear blister canker disease on the perry pear (Pyrus communis) cv. A20. PBCVd was not detected by dot blot hybridization or bioassay in any of the tested species of Amelanchier, Aronia, Cotoneaster, Crataegus, and Pyracantha. However, some species of Chaenomeles, Cydonia, and Sorbus, five out of 13 species of Malus, 15 Pyrus species, and 16 commercial pear cultivars were susceptible to PBCVd, although none developed symptoms. Only in perry pear seedlings did approximately $5 \%$ of the population react to infection with pure PBCVd strains by developing petiole, leaf, or bark necrosis 2 to 3 years (cv. A20) or 3 to 5 months (cv. Fieudière) after inoculation. The selected Fieud 37 and Fieud 110 clones are proposed as PBCVd indicators to replace A20. Results from bioassays on the indicators and from detection by a PBCVd-cRNA probe were essentially in agreement except for some Malus species.
\end{abstract}

Additional keywords: molecular hybridization, Pyrus viroid

In the 1960 s, a perry pear, Pyrus communis cv. A20, was selected at Long Ashton (England) because it clearly exhibited the typical symptoms caused by pear vein yellows virus (PVYV) as well as pear ring pattern mosaic caused by some strains of apple chlorotic leaf spot virus (ACLSV). A20 was introduced at Lanxade (France) in 1966 as an indicator for these two diseases. In 1969, another disorder, pustule bark canker, appeared on A20 plants grafted 2 years before with buds from the pear cultivar Beurré Diel P47A, infected with PVYV and ACLSV but with no evidence of any bark alteration. That was the first indication of the A20 blister canker disease (4), so-called because the symptoms are similar to those of a pear disease described previously (3). Thereafter, cultivar A20 was used to index pear cultivars and to study the new pear bark disorder.

Twenty years later, analysis by polyacrylamide gel electrophoresis (PAGE) led to the discovery of the causal agent of the

\section{Corresponding author: J. C. Desvignes \\ E-mail: s-lanxade@ctifl.fr}

Accepted for publication 20 January 1999.

Publication no. D-1999-0223-02R

(C) 1999 The American Phytopathological Society
A20 blister canker disease, the pear blister canker viroid (PBCVd), a circular RNA of 315 to 316 nucleotides $(1,5,8)$. The bark disorder appeared only on A20 grafted on pear seedlings or quince layers 2 to 3 years after inoculation. Here, we report the results of a search for an alternative indicator that may give a faster reaction, together with a comparison of the biological and molecular approaches for detecting PBCVd.

\section{MATERIALS AND METHODS}

Viroid isolates. In studies conducted some 20 years ago in the Lanxade field plots, about 50 pear cultivars, apparently virus-free after thermotherapy, were indexed on A20 by double grafting. After 2 to 3 years, three of them, P2098T, P1914T, and P10433T, induced on the A20 bark the same pustule canker previously caused by Beurré Diel P47A. These four isolates, particularly P2098T, were used in different studies to identify and characterize the causal agent PBCVd $(1,5,8)$, with the aim of setting up a detection protocol by molecular hybridization (2). In 1993 and 1994, 152 old French varieties collected in different regions were tested by indexing on A20 and by molecular hybridization as indicated below.

Preparation of pure PBCVd strains. Results have been reported from studies on
PBCVd transmission by stem slashing with purified PBCVd obtained by two consecutive PAGE steps consisting of nondenaturing then denaturing conditions $(1,5)$. On the other hand, a second technique referred to as time of contact was used to free PBCVd from accompanying pathogens. For this purpose, bark chips of the P47A cultivar were grafted (one chip per plant, six plants per test) on A20 plants. The grafts were either removed after 2 to 5 days, or left, in the case of the controls. During a 3-year period, the A20 plants were monitored to select those infected by PBCVd but free of other pathogens.

Transmission assays of PBCVd to several pomaceous genera. In our search for a better PBCVd indicator that develops symptoms in a shorter time, we inoculated the P2098T isolate by chip budding onto plants of the following 17 pomaceous species and grew them in the greenhouse: Amelanchier canadensis, A. grandiflora, A. vulgaris, Aronia melanocarpa, Chaenomeles japonica, Cotoneaster franchetii, Crataegus coccinioides, C. marshallii, $C$. monogyna (cv. Fec and San), C. viridis, Cydonia oblonga, Pyronia veitchii, Pyracantha coccinea, Sorbus aucuparia, $S$. aurentiaca, S. mougeotti, and S. pratii. The same P2098T isolate was also inoculated onto 13 Malus species.

Transmission assays of PBCVd to several Pyrus species and varieties. In a first field assay, the P2098T isolate was inoculated onto 16 commercial pear cultivars budded on the Brokmal pear rootstock. Over the next 6 years, the plants were observed for alterations in the bark, foliage, or growth pattern. In a second greenhouse assay, the same PBCVd isolate was inoculated to seedlings or grafts of 15 different Pyrus species that were observed for 2 years to record any emergence of bark canker or leaf necrosis.

Search for differential sensitivity to PBCVd in pear seedling populations. In the first experiment, 105, 54, and 108 seedlings of cv. Fieudière perry pear, A20, and Pyrus serotina, respectively, were inoculated with the isolate P2098T and observed in the greenhouse for 2 years. The second experiment was based on 250 young Fieudière seedlings. Material from 
each plant was propagated by tip-grafting on young seedlings and inoculated with the isolate P2098T. After 1 year in the greenhouse, some of the plants displayed necrosis, and material from the corresponding noninoculated seedlings was multiplied by grafting and by in vitro propagation to confirm and extend these results.

Molecular hybridization. A detailed account of the extraction and hybridization procedures has been published previously (2). Briefly, nucleic acids were extracted from samples $(2 \mathrm{~g})$ of leaves, 3 months or older, collected from different plant parts. At least duplicate analyses were conducted for each genus or species together with the corresponding noninoculated controls grown under the same conditions. Dot blot hybridization was performed with a di- goxigenin-labeled full-length RNA probe complementary to the $\mathrm{PBCVd}$ reference sequence from isolate P2098T (accession no. S46812). Visible hybridization signals were taken as indicative of the viroid presence because no signal was observed with the noninoculated controls.

\section{RESULTS}

Distribution of PBCVd in different pear cultivars. Typical blister cankers on the A20 indicator (Fig. 1) and clear hybridization signals on the membranes were produced by 15 of the 152 old French pear varieties tested. The susceptible varieties included: Besi de Saint Vaast, Beurré Beaumont, B. Durondeau, Bonne Louise, Bronzée d'Enghien, Charles Ernest, Conférence, Epine du Mas, Fondante Maître

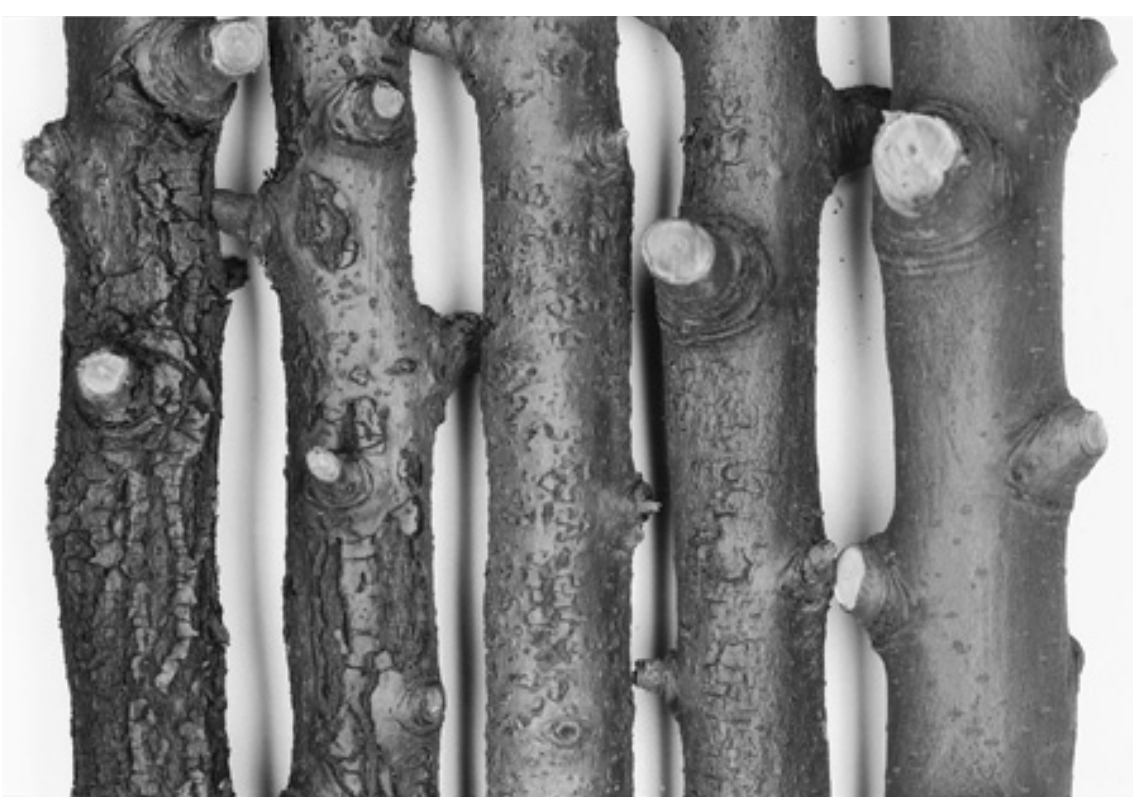

Fig. 1. Blister canker disease on the pear cv. A20 indicator 3 years after inoculation with isolates of different severity of pear blister canker viroid ( $\mathrm{PBCVd})$. At the right, a noninoculated A20 plant.

Table 1. Susceptibility of pomaceous species to pear blister canker viroid (P2098T isolate)

\begin{tabular}{llccc}
\hline Genus & Species & Propagation $^{\mathbf{a}}$ & Hybridization $^{\text {Indexing (A20) }}$ \\
\hline Cotoneaster & franchetii & $\mathrm{Se}$ & - & - \\
Crataegus & coccinioides & $\mathrm{Se}$ & - & - \\
Crataegus & marshallii & $\mathrm{Ga}$ & - & - \\
Crataegus & monogyna cv. Fec & $\mathrm{Ga}$ & - & - \\
Crataegus & monogyna cv. San & $\mathrm{Ga}$ & + & + \\
Crataegus & viridis & $\mathrm{Se}$ & - & - \\
Pyracantha & coccinea & $\mathrm{Se}$ &,-- & - \\
Aronia & melanocarpa & $\mathrm{Se}$ & - & + \\
Sorbus & aucuparia & $\mathrm{Ga}$ & + & + \\
Sorbus & aurentiaca & $\mathrm{Se}$ & + &,++ \\
Sorbus & mougeottii & $\mathrm{Ga}$ and Se &,++ & + \\
Sorbus & pratii & $\mathrm{Se}$ & + & + \\
Chaenomeles & japonica & $\mathrm{Se}$ & + & + \\
Cydonia & oblonga & $\mathrm{Se}$ & + & - \\
Pyronia & veitchii & $\mathrm{Gp}$ & + &,- \\
Amelanchier & canadensis & $\mathrm{Se}$ & - & - \\
Amelanchier & grandiflora & $\mathrm{Ga}$ &,- & + \\
Amelanchier & vulgaris & $\mathrm{Se}$ & - & + \\
\hline
\end{tabular}

${ }^{\text {a }}$ Material was propagated by seed (Se), or by grafting on apple (Ga) or pear (Gp) seedlings.

${ }^{\mathrm{b}}+$, visible signal (hybridization) and/or bark symptoms (bioassay); -, no signal (hybridization), no symptoms (bioassay).

${ }^{c}$ Material from two origins.
d'Ecole, Général de Bonchamps, Muscat d'Hiver, Notaire Lepin, Nouveau Poitou, Roi Humbert, and Seigneur Espéren. The pustule bark cankers were similar, but those induced by some varieties differed consistently in their number and size as if they were caused by different strains. With the four reference isolates, the symptom severity increased from P1914T to P2098T, P10433T, and P47A. The PBCVd cRNA probe derived from isolate $\mathrm{P} 2098 \mathrm{~T}$ was able to detect other PBCVd isolates with similar sensitivity.

Isolation of PBCVd free from other accompanying pathogens. $\mathrm{PBCVd}$ purified by PAGE is able to induce the typical blister cankers on the A20 indicator (1). In addition to this physical procedure, the time of contact approach permitted the separation of ACLSV and PVYV from PBCVd isolate P47A. In this respect, we observed that when the chip inoculum was removed after 2 to 3 days, only PBCVd was transmitted into some of the receptor plants, whereas ACLSV and PVYV needed longer contact times (4 to 5 days).

Susceptibility of some members of different pomaceous genera to infection by PBCVd. The viroid was not detected in the members of the genera Amelanchier, Aronia, Cotoneaster, and Pyracantha that were tested (Table 1). Of the five Crataegus species tested, the viroid was detected only in Crataegus monogyna cv. San. Samples from this species produced a clear hybridization signal and blister cankers when back indexed on A20. This latter result, however, needs further confirmation. By contrast, dot blot hybridization and back indexing on A20 from each of the five inoculated plants from genera Chaenomeles, Cydonia, Pyronia, and Sorbus (Table 1) revealed that they were susceptible, although none expressed any visible symptoms and, consequently, they were disregarded as alternative indicators. On the other hand, back indexing on A20 showed that PBCVd did infect some seedlings of Malus baccata, M. Crimson Brillant, $M$. ioensis, $M$. platycarpa, and $M$. robusta (Table 2), even though molecular hybridizations with extracts from Malus were negative in all cases.

Susceptibility of Pyrus species and varieties to PBCVd infection. The 16 Pyrus species, except $P$. serotina, were susceptible to PBCVd, although they remained symptomless (Table 3). Likewise, we did not observe any PBCVd-induced symptoms on the 16 inoculated commercial pear varieties: Abbé Fetel, Beurré Bosc, B. Diel, B. Giffard, B. Hardy, Comice, Conférence, Delbias, Dr. Jules Guyot, Harrow Sweet, Highland, Louise Bonne, Pierre Corneille, Président Héron, Sérenade, and Williams. All of them, however, were shown to be susceptible to PBCVd by hybridization and indexing assays.

Fieudière 37 and 110, two new PBCVd indicators. In view of these 
negative results, we decided to extend our search for an improved PBCVd indicator to several populations of pear seedlings. In the first experiment, no symptoms appeared on any of the $108 P$. serotina seedlings, while only two of the 54 A20 seedlings exhibited some bark cankers 2 years after indexing. As for the 105 Fieudière seedlings, two died rapidly and four exhibited necrosis of petioles and leaves, and went into decline within months after inoculation. These results suggested that a faster biological indicator for PBCVd may exist within populations of Fieudière seedlings.

In a second experiment, three of 250 Fieudière seedlings, numbers 37,110 , and 186, exhibited necrosis on petioles, leaves, and young shoots 3 to 5 months after inoculation in the greenhouse. To confirm these results, noninoculated material from these three seedlings was grafted onto pear seedling rootstock that was simultaneously inoculated, below the graft, with either pure PBCVd strains (P2098T-12 obtained by PAGE and P47-3d by removing the chip inoculum after a contact period of 3 days) or with different PBCVd, PVYV, and ACLSV isolates. The characteristic PBCVd necrosis appeared after 3 to 4 months on Fieud 37 and Fieud 110 clones, and after 5 months on Fieud 186 (this clone was discarded for future studies). Necrosis began at the base of the petioles and extended to the leaves and bark (Fig. 2). In total, Fieud 37 and Fieud 110 clones were tested with $30 \mathrm{PBCVd}$ isolates, which in all cases induced the typical symptoms, and 22 PBCVd-free sources, which did not induce these symptoms. The same two Fieud 37 and Fieud 110 clones expressed the typical veinclearing and chlorotic mottling when inoculated with PVYV, but no symptoms were observed when they were inoculated with ACLSV. Grafted or in vitro-propagated young plants showed the same symptoms throughout the year in the greenhouse. With the in vitro-propagated plants, the quickest reactions were obtained when the indicators were inoculated 10 to 12 days after a dormant period of 3 months in a cold chamber at 4 to $5^{\circ} \mathrm{C}$, and cut back above the inoculum.

Table 2. Susceptibility of Malus species to pear blister canker viroid (P2098T isolate)

\begin{tabular}{lccc}
\hline Species & Propagation $^{\mathbf{a}}$ & Hybridization & Indexing (A20) \\
\hline M. baccata & $\mathrm{Se}$ & $-\mathrm{b}$ & + \\
M. baccata gracilis & $\mathrm{Ga}$ & - & - \\
M. Crimson Brillant & $\mathrm{Se}$ & - & + \\
M. floribunda & $\mathrm{Se}$ & - & - \\
M. hupehensis & $\mathrm{Se}$ & - & - \\
M. .oensis & $\mathrm{Se}$ & - & + \\
M. micromalus & $\mathrm{Ga}$ & - & - \\
M. platycarpa & $\mathrm{Se}$ &,-- & + \\
M. pumila & $\mathrm{Ga}$ and Se &,-- &,- \\
M. robusta & $\mathrm{Ga}$ and Se & - &,- \\
M. rubifolia & $\mathrm{Ga}$ & - & - \\
M. sieboldii & $\mathrm{Se}$ &,-- &,-- \\
M. yunnanensis & $\mathrm{Ga}$ and Se &
\end{tabular}

a Material was propagated by seed (Se), or by grafting ( $\mathrm{Ga}$ ) on apple seedlings.

${ }^{\mathrm{b}}+$, visible signal (hybridization) and/or bark symptoms (bioassay); -, no signal (hybridization), no symptoms (bioassay).

Table 3. Susceptibility of Pyrus species to pear blister canker viroid (P2098T isolate)

\begin{tabular}{lccc}
\hline Species & Propagation $^{\text {a }}$ & Hybridization & Indexing (A20) \\
\hline P. alpina & $\mathrm{Gp}$ & $+^{\mathrm{b}}$ & + \\
P. amygdaliformis & $\mathrm{Gp}$ and Se &,++ &,++ \\
P. betulifolia & $\mathrm{Gp}$ & + & + \\
P. calleryana & $\mathrm{Gp}^{\mathrm{c}}$ &,++ &,++ \\
P. canescens & $\mathrm{Gp}$ and Se &,++ &,+ \\
P. communis cv. B. Bosc & $\mathrm{Gp}$ & + & + \\
P. communis cv. Williams & $\mathrm{Gp}$ & + & + \\
P. lindleti & $\mathrm{Gp}$ & + & + \\
P. malifolia & $\mathrm{Gp}$ & - & + \\
P. michauxii & $\mathrm{Gp}$ & + & + \\
P. nivalis & $\mathrm{Gp}$ & + & + \\
P. persica & $\mathrm{Gp}$ & + & + \\
P. pollveria & $\mathrm{Gp}$ &,++ & + \\
P. salicifolia & $\mathrm{Gp}$ and Se & - & + \\
P. serotina & $\mathrm{Gp}$ & + & + \\
P. sinaica & $\mathrm{Gp}$ & + & + \\
Pyrus $\times$ Malus hybrid & $\mathrm{Gp}$ & + & +
\end{tabular}

\footnotetext{
${ }^{a}$ Material was propagated by seed (Se), or by grafting (Gp) on pear seedlings.

${ }^{\mathrm{b}}+$, visible signal (hybridization) and/or bark symptoms (bioassay); -, no signal (hybridization), no symptoms (bioassay).

${ }^{\mathrm{c}}$ Material from two origins.
}

\section{DISCUSSION}

The distribution and pathogenic effects of PBCVd have probably been underestimated because (i) pear and quince cultivars are generally tolerant, and (ii) when they are sensitive, the infected trees decline quickly or exhibit bark cankers as if they were infected by bacteria or fungi, which are then presumed to be the causal agent. We know PBCVd has been widespread in France and other parts of Europe for a long time, because it was detected in several old local varieties that are no longer propagated (J. C. Desvignes, unpublished data). Some of the old local varieties may have been sensitive to $\mathrm{PBCVd}$, but it was not possible to examine more than 200 varieties at that time.

In this study, we analyzed by bioassay on cv. A20 and by molecular hybridization 152 old French pear varieties and found that approximately $10 \%$ were infected by PBCVd. Symptom severity on A20 was different among isolates that in the case of the PBCVd reference isolates P2098T, P1914T, and P47A might be explained on the basis of their sequence heterogeneity $(1,8)$. By means of these two detection methods, we also observed that the 16 Pyrus species studied (except $P$. serotina), species of some pomaceous genera, seedlings of some Malus species, and a Pyrus $\times$ Malus hybrid were susceptible to PBCVd, in all cases resulting in symptomless infections (Tables 1 to 3 ). Therefore, PBCVd is not Pyrus-specific and appears to have nearly the same host range as the type member of the viroid genus to which it belongs, apple scar skin viroid $(6,7)$, which can infect both Malus and Pyrus genera $(9,10)$, as well as Chaenomeles, Cydonia,

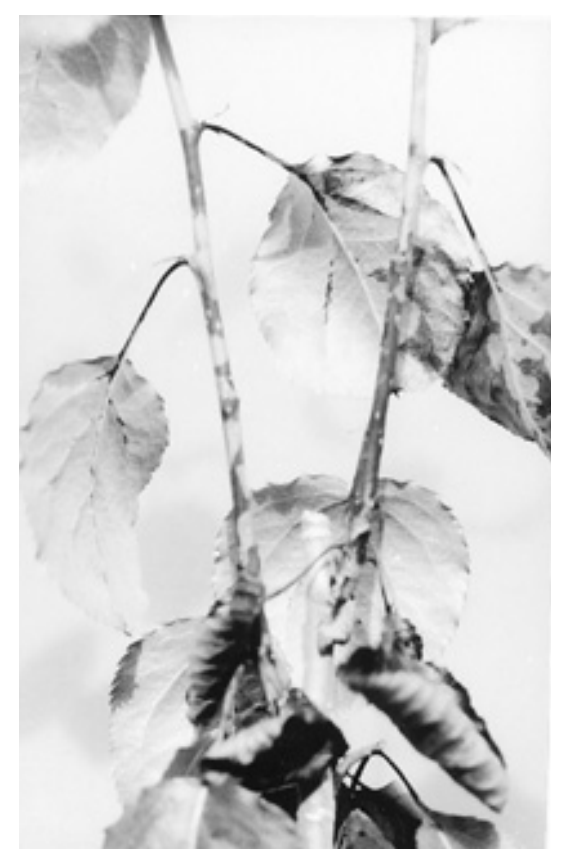

Fig. 2. Necrosis on the pear cv. Fieud 37 indicator 3 to 4 months after inoculation with an isolate of pear blister canker viroid (PBCVd). 
Pyronia, and Sorbus (J. C. Desvignes, unpublished data).

We observed a close correlation between the two detection approaches evaluated here except for some Malus species, in which the viroid was undetected by dot blot hybridization, probably because its concentration was below the limit of detection. We propose molecular hybridization with a PBCVd-cRNA probe for a rapid detection of the pathogen in leaves, when inspecting material exchanges and the introduction of new cultivars. We also recommend replacing A20 with the new indicators Fieud 37 and Fieud 110, grown in a greenhouse, to confirm results obtained by hybridization. Grafted or in vitro-propagated material will allow observation of the development of the disease and its epidemiology, as well as examination of budwood and apple cultivars for latent infections by PBCVd. The two new PBCVd indicators can be obtained from the Lanxade Center on request.
ACKNOWLEDGMENTS

We thank A. Ahuir for excellent technical assistance. This work was partially supported by contract AIR3CT93-1567 from the European Commission (to J. C. D. and R. F.) and by grant PB95-0139 from the Dirección General de Investigación Científica y Técnica of Spain (to R. F.).

\section{LITERATURE CITED}

1. Ambrós, S., Desvignes, J. C., Llácer, G., and Flores, R. 1995. Pear blister canker viroid: Sequence variability and causal role in pear blister canker disease. J. Gen. Virol. 76:26252629.

2. Ambrós, S., Desvignes, J. C., Llácer, G., and Flores, R. 1995. Peach latent mosaic and pear blister canker viroids: Detection by molecular hybridization and relationships with specific maladies affecting peach and pear trees. Acta Hortic. 386:519-521.

3. Cropley, R. 1960. Pear blister canker: A virus disease. Annu. Rep. East Malling Res. Stn. 1959. 43:104.

4. Desvignes, J. C. 1970. Les maladies à virus du poirier et leur détection. Ctifl Doc. 26:112.

5. Flores, R., Hernández, C., Llácer, G., and Desvignes, J. C. 1991. Identification of a new viroid as the putative causal agent of pear blister canker disease. J. Gen. Virol. 72:11991204.

6. Flores, R., Randles, J. W., Bar-Joseph, M. and Diener, T. O. 1998. A proposed scheme for viroid classification and nomenclature. Arch. Virol. 143:623-629.

7. Hashimoto, J., and Koganezawa, H. 1987. Nucleotide sequence and secondary structure of apple scar skin viroid. Nucleic Acids Res. 15:7045-7052.

8. Hernández, C., Elena, S. F., Moya, A., and Flores, R. 1992. Pear blister canker viroid is a member of the apple scar skin viroid subgroup (apscaviroids) and also has sequence homologies with viroids from other subgroups. J. Gen. Virol. 73:2503-2507.

9. Osaki, H., Kudo, A., and Ohtsu, Y. 1996 Japanese pear fruit dimple disease caused by apple scar skin viroid (ASSVd). Ann. Phytopathol. Soc. Jpn. 62:379-385.

10. Zhu, S. F., Hadidi, A., Hammond, R. W., Yang, X., and Hansen, J. A. 1995. Nucleotide sequence and secondary structure of pome fruit viroids from dapple apple diseased apples, pear rusty skin diseased pears and apple scar skin symptomless pears. Acta Hortic. 386:554-559. 